\title{
Vigilância ambiental em saúde: veiculação das ações de prevenção e promoção da
}

\section{saúde}

\author{
Environmental health surveillance: dissemination of prevention and health promotion actions \\ Vigilancia de salud ambiental: difusión de acciones de prevención y promoción de la salud
}

Recebido: 14/12/2021 | Revisado: 22/12/2021 | Aceito: 15/01/2022 | Publicado: 16/01/2022

\author{
Maria Assunta Busato \\ ORCID: https://orcid.org/0000-0003-0043-7037 \\ Universidade Comunitária da Região de Chapecó, Brasil \\ E-mail: assunta@unochapeco.edu.br \\ Ícaro Moraes Colella \\ ORCID: https://orcid.org/0000-0001-9425-0198 \\ Universidade Comunitária da Região de Chapecó, Brasil \\ E-mail: icarocolella21@gmail.com \\ Ariane Fátima Deggeroni \\ ORCID: https://orcid.org/0000-0001-8219-6083 \\ Universidade Comunitária da Região de Chapecó, Brasil \\ E-mail: arianedeggeroni@gmail.com \\ Junir Antônio Lutinski \\ ORCID: https://orcid.org/0000-0003-0149-5415 \\ Universidade Comunitária da Região de Chapecó, Brasil \\ E-mail: junir@unochapeco.edu.br
}

\begin{abstract}
Resumo
A Vigilância Ambiental em Saúde (VAS) tem como competência institucional a gestão do sistema nacional de vigilância ambiental, com o propósito de exercer a vigilância dos fatores de risco ambientais que acometem a saúde da população. Objetivo deste estudo é analisar a veiculação das ações de prevenção de agravos e de promoção da saúde realizadas pela VAS, no período de 2008 a 2017, no município de Chapecó, SC. Os documentos analisados foram os produzidos e divulgados pela Assessoria de Imprensa do município, disponibilizados em Digital Versatile Disc (DVD) com clipagens das ações veiculadas pela imprensa. O estudo analisou 681 matérias midiáticas. A maioria foi matéria jornalística $(40,6 \%)$, seguida de fotos de registros das ações $(16,7 \%)$ e matérias de rádio $(13,8 \%)$. Apresenta os setores envolvidos e os eixos temáticos das ações e campanhas midiáticas representadas, especialmente, por informações epidemiológicas, de educação em saúde, de sensibilização da comunidade e visitas domiciliares. A maior intensidade de publicações foi entre dezembro e abril, devido à infestação pelo mosquito Aedes aegypti e o risco para a transmissão da dengue. Os meios jornalísticos são fortes aliados como estratégia comunicativa para que ações em saúde tenham êxito. Evidencia-se a intersetorialidade como indispensável para a efetividade das ações de promoção da saúde e na prevenção de agravos. Sobretudo, aproximam os serviços de saúde da população, oportunizam a participação social nas ações e contribuem para transparência na prestação do serviço.
\end{abstract}

Palavras-chave: Comunicação em saúde; Vigilância em saúde; Educação em saúde.

\begin{abstract}
Environmental Health Surveillance (VAS) has an institutional competence of management of the national environmental surveillance system and with the purpose of exercising surveillance of environmental risk factors that affect the population's health. Objective this study is to analyze the dissemination of health prevention and health promotion actions carried out by VAS, from 2008 to 2017, in the municipality of Chapecó, SC. The documents analyzed were those produced and disseminated by the Press Office of the municipality, made available on Digital Versatile Disc (DVD) with clips of the actions published by the press. The study analyzed 681 media articles, most of which were journalistic articles $(40.6 \%)$, followed by photos of stock records $(16.7 \%)$ and radio articles $(13.8 \%)$. It presents the sectors involved and the thematic axes of the actions and media campaigns represented, especially by epidemiological information, health education, community awareness and home visits. The highest intensity of publications was between December and April, due to the infestation by the Aedes aegypti mosquito and the risk for dengue transmission. Journalistic media are strong allies as a communicative strategy for health actions to be successful. In the same way, intersectorality is shown to be indispensable for the effectiveness of health promotion actions and disease prevention. They bring health services closer to the population, provide social participation in actions and contribute to transparency in service provision.
\end{abstract}

Keywords: Health communication; Health surveillance; Health education. 


\begin{abstract}
Resumen
La Vigilancia en Salud Ambiental (VAS) tiene la competencia institucional de gestionar el sistema nacional de vigilancia ambiental, con el propósito de ejercer la vigilancia de los factores de riesgo ambiental que afectan la salud de la población. El objetivo de este estudio es analizar la transmisión de las acciones de prevención de enfermedades y promoción de la salud realizadas por la VAS, de 2008 a 2017, en la ciudad de Chapecó, SC. Los documentos analizados fueron los elaborados y difundidos por la Oficina de Prensa del municipio, disponibles en un Disco Versátil Digital (DVD) con recortes de las acciones publicadas por la prensa. El estudio analizó 681 materias mediáticas. La mayoría eran historias periodísticas (40,6\%), seguidas de fotos de archivo (16,7\%) y noticias de radio $(13,8 \%)$. Presenta los sectores involucrados y los ejes temáticos de acciones y campañas mediáticas representadas, especialmente, por la información epidemiológica, la educación en salud, la sensibilización comunitaria y las visitas domiciliarias. La mayor intensidad de publicaciones fue entre diciembre y abril, debido a la infestación por el mosquito Aedes aegypti y al riesgo de transmisión del dengue. Los medios periodísticos son fuertes aliados como estrategia comunicativa para el éxito de las acciones de salud. La intersectorialidad se evidencia como fundamental para la efectividad de las acciones de promoción de la salud y prevención de enfermedades. Sobre todo, acercan los servicios de salud a la población, brindan espacios de participación social en las acciones y contribuyen a la transparencia en la prestación del servicio.
\end{abstract}

Palabras clave: Comunicación en salud; Vigilancia de la salud; Educación para la salud.

\title{
1. Introdução
}

No ano 2000 a Fundação Nacional de Saúde (FUNASA), órgão do Ministério da Saúde, por meio do Decreto ${ }^{\circ}$ 3.450/2000, iniciou oficialmente no Brasil o processo de implantação da Vigilância Ambiental em Saúde (VAS) no âmbito do Sistema Único de Saúde (SUS). Estabeleceu a implantação do sistema nacional de vigilância ambiental em todo território nacional com o propósito de exercer a vigilância dos fatores de risco ambientais que acometem a saúde da população (Brasil, 2002).

A VAS possibilita a estruturação de um serviço que atenda às diretrizes na área da saúde relacionadas à saúde e ambiente. A Lei 8.080/90 (lei do SUS), Art. 6º , estabelece no campo de atuação, a execução de ações de vigilância sanitária, vigilância epidemiológica, saúde do trabalhador e assistência terapêutica integral (Brasil, 1990). Em 2003, ocorreu a criação da Secretaria de Vigilância em Saúde (SVS) e, com esta, o serviço voltado à saúde ambiental. Este serviço foi incorporado ao Ministério da Saúde para atuar de forma integrada com as demais vigilâncias, no âmbito da SVS. Essa integração das vigilâncias foi se fortalecendo nas três esferas de governo, impulsionada pela relevância das doenças e agravos à saúde, foco das ações de prevenção, e pela urgência de respostas rápidas diante de emergências de saúde pública. Ademais, segundo Silva et al. (2021), o diálogo entre setores, a Intersetorialidade, resulta da articulação entre pessoas de áreas diversas, com saberes, poderes e vontades variadas, visando resolver problemas complexos.

Destaca-se que a VAS possui um caráter integrador inter e intrasetorial, o que facilita a criação de vínculos e de articulação com todas as outras formas de vigilância, definidas institucionalmente e presentes nos serviços de saúde ${ }^{4}$. No âmbito do SUS, a VAS constitui-se no conjunto de serviços e ações que proporcionam conhecimento e a detecção de fatores de risco do meio ambiente que interferem na saúde humana, com a finalidade de identificar as medidas de prevenção e controle dos fatores de risco ambientais relacionados às doenças ou a outros agravos à saúde (Busato et al., 2018). O setor está dividido em subáreas de atuação, Coordenação de Vigilância e Controle dos Fatores de Risco Biológicos (COFAB): vetores, hospedeiros, reservatórios, e Coordenação de Vigilância e Controle dos Fatores de Risco Não Biológicos (CONAB): água, ar, solo, desastres e contaminantes (Brasil, 2002).

Após mais de uma década da implantação da VAS, ainda necessita de avanços e mudanças, especialmente quando se vivencia cenários de incertezas decorrentes das mudanças ambientais em nível local e global. Também é necessário aproximar conceitos e ações da Vigilância em Saúde para os profissionais das equipes de saúde da família de modo que isto desenvolva uma reflexão sobre as ações de saúde nos seus territórios (Costa et al., 2021). Nessa perspectiva, as discussões das condições 
do ambiente e sua relação com a saúde humana podem ser consideradas contemporâneas, pois está em cada momento construindo conceitos e compreensões (Busato et al., 2013).

Um dos grandes desafios da VAS é possuir uma identidade própria, que ainda está em processo de consolidação. Em nível nacional, a lógica de planejamento e gestão segue uma abordagem diferente dos municípios, dificultando a consolidação desse campo da vigilância em saúde no SUS (Bezerra, 2017). Outra dificuldade para a efetivação/consolidação da VAS, afirmada por (Silva et al., 2021), está direcionada na necessidade de reestruturação da das ações de vigilância dos estados e municípios, bem como a formação de equipes multidisciplinares com capacidade de articulações interdisciplinares e intersetoriais.

Nesse contexto, a comunicação é um forte aliado para proporcionar a articulação entre saúde e ambiente, [...] "assunto complexo e controverso que, apesar de todos os esforços, continua se afigurando de forma difusa, imprecisa e muito mais retórica do que consistente, tanto na teoria como na prática" (Gómez-Minayo \& Minayo, 2006). Daí a importância da comunicação em saúde e da divulgação de ações e campanhas de prevenção e promoção da saúde.

Nos últimos anos percebe-se um importante aumento de publicações, documentários e campanhas de publicidade sobre as questões ambientais e de saúde em veículos como emissoras de rádios, televisão e jornais. Considerando esses veículos como importantes fontes de informação para a população, o papel desses meios é decisivo nos processos de formação de opinião sobre a relação da saúde com o ambiente, bem como na mobilização da participação social e no empoderamento da sociedade para autocuidado em saúde.

As questões de saúde-doença e meio ambiente pautam diálogos nos mais diversos meios de comunicação. Para Pessoni e Passaro (2021), os setores de saúde devem fazer uso de uma comunicação estratégica, integrada e multimídia, guiada pelos princípios do SUS para atingir com mais eficiência e eficácia os públicos. Isso vale para profissionais de diferentes áreas, incluindo os de comunicação. Também, integrar comunicação, saúde e meio ambiente, no âmbito da vigilância, implica superar a lógica disciplinar isolada). Neste sentido, as Diretrizes Curriculares Nacionais (DCN) para os cursos de comunicação reforçam a necessidade de conteúdos que associem teoria e prática. As DCN demandam "conteúdos teórico-conceituais; conteúdos analíticos e informativos sobre a atualidade; conteúdos de linguagens, técnicas e tecnologias midiáticas e conteúdos ético-políticos" (Brasil, 2001), a exemplo da divulgação das ações e campanhas da VAS.

Diante desse cenário e do reconhecimento da importância da ação conjunta de diferentes setores, este estudo teve por objetivo analisar a veiculação das ações de prevenção de agravos e de promoção da saúde realizadas pelo serviço de Vigilância Ambiental em Saúde, relativas ao período de 2008 a 2017, no município de Chapecó, Santa Catarina. Esse município estruturou o serviço de VAS no ano de 2008, impulsionado pela implantação do Plano Municipal de Controle à Dengue e Febre Amarela (PMCD), face ao aumento dos índices de infestação por vetores na região (Lutinski et al., 2018).

\section{Metodologia}

Este estudo é do tipo descritivo com abordagem quantitativa, assim caracterizado pois descreve os fatos observados sem causar interferência neles (Santos et al., 2018). O local de estudo foi o município de Chapecó, fundado no ano de 1917, localizado sob as coordenadas geográficas: Latitude: $27^{\circ} 05^{\prime} 48^{\prime \prime}$ Sul; Longitude: $52^{\circ} 37^{\prime} 07^{\prime \prime}$ Oeste, a $550 \mathrm{~km}$ da capital, Florianópolis. O clima da região é subtropical e as temperaturas médias anual do Oeste de Santa Catarina é inferior a $20^{\circ} \mathrm{C}$. No verão, as temperaturas máximas raramente ultrapassam os $38^{\circ} \mathrm{C}$ e, no inverno, as temperaturas mínimas são inferiores a zero grau (IBGE, 2021). O município é a principal cidade do oeste catarinense e é um polo agroindustrial regional e da mesorregião oeste. Também é considerado referência nacional nas atividades de suinocultura e avicultura, e referência regional em prestação de serviços na área da saúde (Chapecó, 2015). 
A coleta de dados foi realizada junto à Assessoria de Imprensa do município de Chapecó, em todos os documentos de divulgação produzidos que registram as ações desempenhadas pela VAS. A prefeitura de Chapecó disponibilizou, mediante solicitação e aprovação formal via ofício, três DVDs com clipagens das ações veiculadas pela imprensa no período de 2008 a 2017, relativas às ações/estratégias de prevenção e promoção da saúde.

As informações foram organizadas em planilhas, por produto midiático sendo, matérias em jornais locais, rádio e televisão, além dos conteúdos produzidos pela Assessoria de Imprensa da Prefeitura que correspondem a itens de publicidade, tais como banners, flyers e cartilhas. Os registros midiáticos foram categorizados nas seguintes categorias: mês e ano da publicação, setores envolvidos, recurso de divulgação, focos da campanha, temáticas e fontes da matéria.

A partir de uma análise de conteúdo, as variáveis foram organizadas em um banco de dados no software Excel for Windows (Microsoft). Foram exploradas quanto às frequências, agrupadas e comparadas quanto à média e desvio padrão. As medianas foram comparadas utilizando-se o teste não paramétrico de Kruskal-Wallis. A opção por este teste deu-se em função da natureza não paramétrica das distribuições, aferidas pelo teste de Shapiro-Wilk. Os testes foram realizados com auxílio do software Past (Hammer et al., 2001).

\section{Resultados e Discussão}

O estudo analisou 681 matérias midiáticas referente ao período de 2008 a 2017, sendo que a maioria foi matéria jornalística (40,6\%), seguido de fotos de registros de ações (16,7\%) e matérias de rádio (13,8\%).

A maior parte das publicações foi feita na forma de matérias jornalísticas em jornais impressos, sejam elas a partir de releases da prefeitura ou materiais clipados de produções de jornais. Destaca-se, também, a atuação radiofônica com entrevistas de especialistas e spots voltados para a mobilização e sensibilização da comunidade (Tabela 1).

Possivelmente, existam mais matérias de televisão produzidas no período, entretanto, devido à dificuldade de armazenar e ter acesso a esses materiais eles aparecem apenas 20 vezes ao longo dos anos. Já os materiais publicitários como post na internet, outdoors, banners e flyers informativos aparecem em sua totalidade. 
Tabela 1. Recursos de divulgação utilizados nas ações de prevenção de agravos e promoção da saúde, realizadas pelo serviço de Vigilância Ambiental em Saúde de Chapecó, divulgadas e registradas pela Assessoria de Imprensa do município, no período de 2008 a 2017.

\begin{tabular}{|c|c|c|c|c|c|c|}
\hline Recursos de divulgação & Total & Média & $\begin{array}{l}\text { Desvio } \\
\text { padrão }\end{array}$ & Percentual & Significância & \\
\hline Matéria jornalística & 277 & 27,70 & 44,66 & 40,68 & \multirow{16}{*}{$\begin{array}{r}\mathrm{Hc}=14,12 \\
0,18\end{array}$} & \multirow{15}{*}{$\mathrm{p}=$} \\
\hline Fotos & 114 & 22,80 & 36,65 & 16,74 & & \\
\hline Matéria de rádio & 94 & 10,44 & 11,93 & 13,80 & & \\
\hline Sugestão de pauta & 45 & 5,00 & 4,76 & 6,61 & & \\
\hline Matéria de televisão & 20 & 3,33 & 2,98 & 2,94 & & \\
\hline Charges & 14 & 4,67 & 3,09 & 2,06 & & \\
\hline Entrevistas & 10 & 2,00 & 0,63 & 1,47 & & \\
\hline Arte para jornal impresso & 8 & 8,00 & 0,00 & 1,17 & & \\
\hline Post na Internet & 7 & 2,33 & 1,89 & 1,03 & & \\
\hline Outdoor & 5 & 1,67 & 0,94 & 0,73 & & \\
\hline Banner & 5 & 1,67 & 0,94 & 0,73 & & \\
\hline Anúncio impresso & 4 & 2,00 & 1,00 & 0,59 & & \\
\hline Apresentação de Plano de Ações & 3 & 3,00 & 0,00 & 0,44 & & \\
\hline Flyer informativo & 3 & 1,50 & 0,50 & 0,44 & & \\
\hline Outros & 72 & 7,20 & 5,38 & 10,57 & & \\
\hline Total & 681 & & & 100 & & \\
\hline
\end{tabular}

Fonte: Assessoria de Imprensa município de Chapecó-SC.

Os meios de comunicação têm um impacto transformador, pelo potencial de alcance que passaram a ter. Inicialmente, o rádio foi o meio mais empregado, porém, a televisão tornou-se a principal fonte de informação pela reprodução de imagens e áudios de forma instantânea e, posteriormente como entretenimento da população. Assim, as campanhas tornaram-se a principal estratégia de publicidade e propaganda com esse recurso (Albarado, 2019).

A intensidade de matérias veiculadas pelos jornais, rádios e TVs locais e regionais, compreendem mais de $64 \%$ das divulgações, o que indica importante forma de atingir e sensibilizar a população do município. A gama de dispositivos e recursos utilizados na divulgação dessas informações fazem parte das estratégias de comunicação, que surgiram a partir de estudos que relacionam educação e saúde. Nesse conjunto, surge o termo comunicação em saúde, preceito que refere

“[...] a processos dialógicos e à utilização de estratégias comunicacionais que respeitam os direitos à informação, à educação e à saúde. Sua finalidade inclui a prevenção de enfermidades, o incentivo à cidadania por meio da participação social, a transparência na gestão da saúde, bem como a promoção da melhoria da qualidade de vida das pessoas em seus diferentes contextos sociais, por meio de relações interpessoais, da mídia e do conhecimento (Albarado, 2019).

É preciso considerar algumas características para a comunicação ser efetiva. Sendo assim, as mídias devem apresentar uma boa estratégia de comunicação, divulgação e implantação da informação, para que atinja a população. Considerando que esta mensagem chegue ao indivíduo, ainda é preciso que ela seja entendida da forma adequada. Após essas etapas, o indivíduo poderá ou não executar a ação educativa e mudar o seu comportamento adotando cuidados com o ambiente e a saúde. Contudo, o desafio da comunicação é informar, reduzir e prevenir doenças. A informação partir dos meios massivos de comunicação (jornais, revistas, rádio, televisão) tem um papel fundamental visto que participa ativamente do processo de informação em saúde (Villela, 2016). 
A VAS do município de Chapecó contou com o apoio intersetorial nas ações desenvolvidas. Foram 29 setores dos serviços públicos municipais, organizações ou instituições envolvidas nas ações e registradas nas divulgações (Tabela 2). As parcerias com outras instituições demonstram uma atuação para além da esfera pública. Essas parcerias envolveram escolas, universidades, polícia militar, bombeiros, entre outras, e reforçam a cooperação na prevenção e promoção da saúde no município.

Tabela 2 - Setores envolvidos nas ações da Vigilância Ambiental em Saúde de Chapecó, divulgadas e registradas pela Assessoria de Imprensa do município, no período de 2008 a 2017.

\begin{tabular}{llllll}
\hline Setores envolvidos & Total & Média & $\begin{array}{l}\text { Desvio } \\
\text { padrão }\end{array}$ & Percentual & Significância \\
\hline Prefeitura Municipal & 412 & 41,20 & 43,94 & 33,33 \\
Secretaria de Saúde & 309 & 30,90 & 36,75 & 25,00 & 16,50 \\
Vigilância Ambiental em Saúde & 204 & 20,40 & 21,34 & 4,45 \\
Vigilância Epidemiológica & 55 & 5,50 & 12,97 & 3,40 \\
Secretaria da Educação & 42 & 4,20 & 7,14 & 2,10 \\
Universidades & 26 & 2,60 & 3,53 & 1,94 \\
Vigilância Sanitária & 24 & 2,40 & 5,55 & 1,62 \\
Vigilância em Saúde & 20 & 2,00 & 4,73 & 1,13 \\
Escolas & 14 & 1,40 & 4,20 & 0,001 \\
Rádios locais & 11 & 1,10 & 1,22 & 0,89 \\
Jornais locais & 8 & 0,80 & 2,09 & 0,65 \\
Secretaria de Serviços Urbanos & 5 & 0,50 & 1,20 & 0,40 \\
Secretaria de Estado da Saúde & 4 & 0,40 & 1,20 & 0,32 \\
Polícia militar & 2 & 0,20 & 0,40 & 0,16 \\
Corpo de Bombeiros & 2 & 0,20 & 0,40 & 0,16 \\
Polícia militar ambiental & 1 & 0,10 & 0,30 & 0,08 \\
Outros & 97 & 9,70 & 15,01 & 7,85 \\
\hline Total & $\mathbf{1 2 3 6}$ & & & $\mathbf{1 0 0}$ \\
\hline
\end{tabular}

Fonte: Assessoria de Imprensa município de Chapecó-SC.

A intersetorialidade parte da lógica de cooperação de Instituições e atores sociais por um bem comum e [...] é definida como um modo de gestão (o que) desenvolvido por meio de processo sistemático de (como) articulação, planejamento e cooperação entre os distintos (com quem) setores da sociedade e entre as diversas políticas públicas para atuar sobre (para que) os determinantes sociais (Akerman, 2014). A importância do trabalho intersetorial, Ventura, Alencar, Araújo e Pinheiro (2019), ao referenciar Rodrigues (2010), rompe os limites em busca da construção de um espaço comunicativo que possibilita a resolução de problemas complexos.

As universidades revelam um perfil participativo e preocupado com a temática saúde. Ao longo dos anos, as instituições educacionais participaram de ações em conjunto com o setor da VAS, sendo essenciais nas atividades de vigilância epidemiológica e ambiental, palestras e mutirões envolvendo estudantes e professores, junto à comunidade. As emissoras de rádio e jornais locais, por sua vez, foram além da divulgação dos releases produzidos pela VAS, através da assessoria de imprensa da prefeitura. Esses veículos realizaram a produção de conteúdo próprio, em sua maioria, com a participação de algum integrante especialista da VAS a fim de divulgar ações ou campanhas de sensibilização. 
As informações em saúde (epidemiológicas e entomológicas), alvo da atuação da VAS, caracterizadas por número de casos, focos, estatísticas históricas e atualizações, aparecem 506 (41,92\%) vezes nos assuntos tratados e, em sua maioria, são referentes à dengue, enfermidade que tem sido recorrente no município, nos últimos anos, especialmente na epidemia de 2016. A Educação em Saúde se destaca das demais temáticas, juntamente com as Informações Epidemiológicas. Os 252 (20,88\%) materiais descritos como Educação em Saúde, foram extraídos de produtos e ações que, além de trazer dados periódicos da epidemia, explicam e educam o consumidor da produção (Tabela 3).

Tabela 3 - Eixos temáticos das ações da Vigilância Ambiental em Saúde de Chapecó, divulgadas e registradas pela Assessoria de Imprensa do município, no período de 2008 a 2017.

\begin{tabular}{|c|c|c|c|c|c|c|}
\hline Eixos temáticos & Total & Média & $\begin{array}{l}\text { Desvio } \\
\text { padrão }\end{array}$ & Percentual & Significância & \\
\hline Informações epidemiológicas & 506 & 50,60 & 44,60 & 41,92 & \multirow{12}{*}{$\begin{array}{l}\mathrm{Hc}=29,38 \\
<0,001\end{array}$} & \multirow{11}{*}{$\mathrm{p}$} \\
\hline Educação em saúde & 252 & 25,20 & 32,16 & 20,88 & & \\
\hline Sensibilização da população & 172 & 17,20 & 31,78 & 14,25 & & \\
\hline Visitas domiciliares & 106 & 10,60 & 15,23 & 8,78 & & \\
\hline Mobilização da comunidade & 64 & 6,40 & 6,70 & 5,30 & & \\
\hline Saneamento básico (RSU) & 32 & 3,20 & 3,76 & 2,65 & & \\
\hline Sala de situação & 13 & 1,30 & 2,33 & 1,08 & & \\
\hline Entrevistas & 13 & 1,30 & 1,19 & 1,08 & & \\
\hline Planejamento & 15 & 1,50 & 2,50 & 1,24 & & \\
\hline Capacitação de ACE & 8 & 0,80 & 1,54 & 0,66 & & \\
\hline Outros & 26 & 5,20 & 6,83 & 2,15 & & \\
\hline Total & 1207 & & & 100,00 & & \\
\hline
\end{tabular}

Fonte: Assessoria de Imprensa município de Chapecó-SC.

As categorizações que resultaram em Educação em Saúde foram: cartilhas educativas que explicam como prevenir a proliferação do mosquito Aedes aegypti Linnaeus, 1762, visitas às escolas, peça de teatro voltado ao público infantil, produção de livros e, também, a articulação das visitas domiciliares devido à resistência às visitas dos Agentes de Combate a Endemias (ACE) em suas residências. Sensibilização da população, seja pelas atividades dos ACE, seja pelas atividades realizadas com setores da sociedade ou com outros setores públicos, bem como a mobilização da sociedade, representou um importante número de ações desenvolvidas pela VAS e divulgadas pela mídia. O complemento entre a realização da ação e sua divulgação ampliam a possibilidade de sensibilização da população para os cuidados do ambiente, a prevenção de doenças e a promoção da saúde.

A série histórica das ações (Figura 1) foi formada a partir de duas categorizações: número de ações e temáticas abordadas. Com relação à frequência de ações foi possível observar um leve crescimento nos primeiros anos de implantação da VAS, ampliando de três ações identificadas e publicizadas em 2008, para 57 em 2010. É notável o elevado número de ações em 2011, ano relativo à primeira confirmação de um caso de dengue no estado de Santa Catarina, o que explica o maior número de publicações sobre essa temática, à época.

Nos anos posteriores a 2011, a média de publicações variou de 25 a 46 entre os anos de 2012 a 2017. Essa característica de periodicidade leva a acreditar num estabelecimento da importância da temática nos diversos meios de comunicação, e também, da relevância dessa divulgação para uma maior sensibilização da população, já que a maior parte dos produtos midiáticos gerados tiveram esse objetivo.

A frequência dos temas abordados no período oscilou consideravelmente, sendo que em 2011 e na epidemia de 
dengue de 2015 e 2016 foram realizadas ações ampliando os eixos temáticos. Evidencia-se que em períodos de picos endêmicos/epidêmicos, são realizados maiores investimentos de planejamento e ações e, consequentemente, maior divulgação de campanhas educativas.

Figura 1 - Frequência de ações e temas abordados nas atividades desenvolvidas pela Vigilância Ambiental em Saúde de Chapecó, divulgadas e registradas pela Assessoria de Imprensa do município, no período de 2008 a 2017.

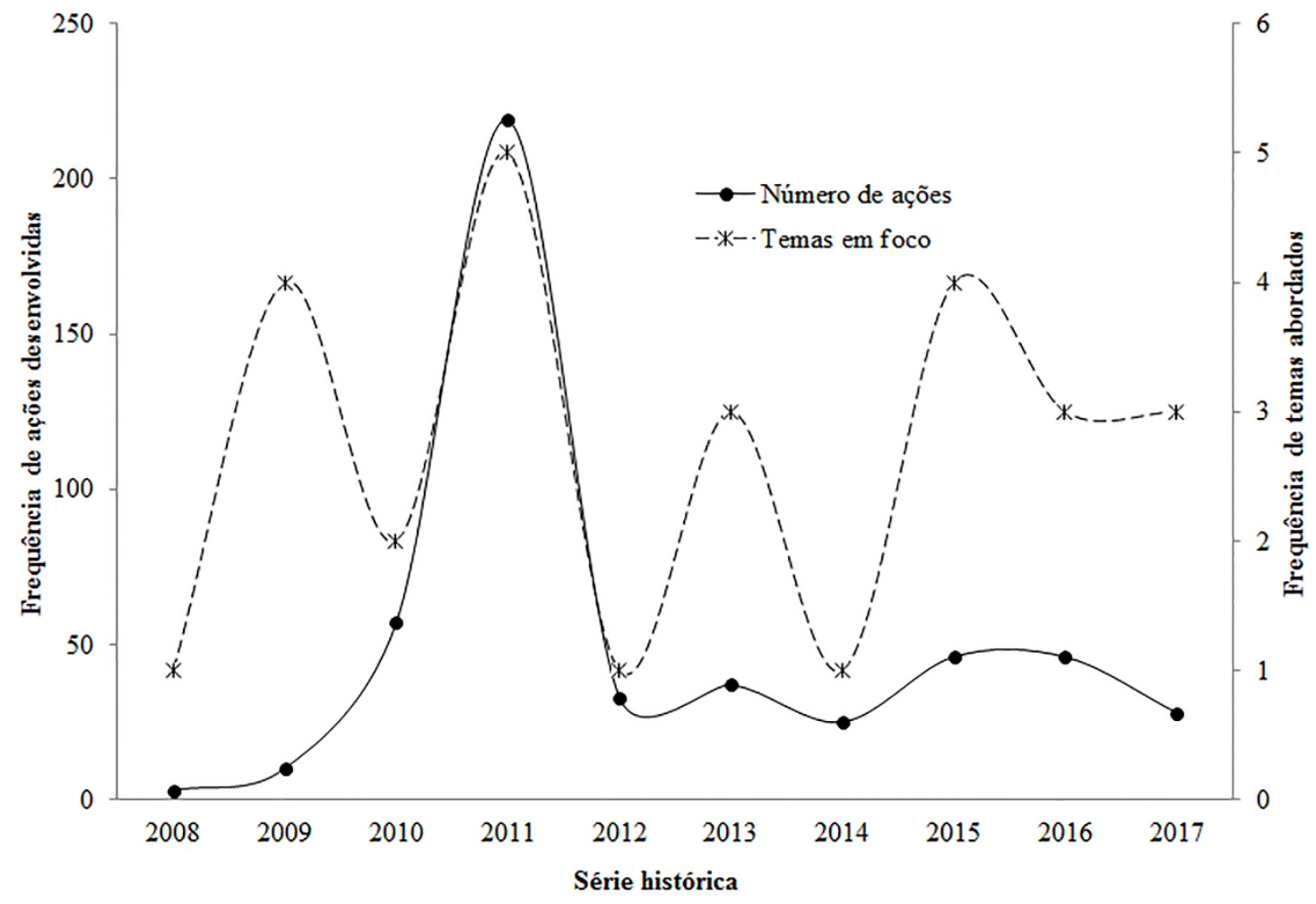

Fonte: Autores.

Para garantir uma maior efetividade, a comunicação dirigida deve ser adotada cotidianamente. Ela tem por finalidade transmitir e conduzir informações, estabelecendo uma comunicação orientada e frequente com o público identificado (Montoro, 2008). Naturalmente, campanhas e ações desenvolvidas pela VAS para a promoção e prevenção da saúde no município de Chapecó ocorrem durante todo o ano. Entretanto, vale ressaltar, que é no período de verão que a dengue se prolifera ainda mais, devido às condições climáticas, que facilitam a reprodução do mosquito. Nesse contexto, o período em que mais foram desenvolvidas ações e divulgação voltadas para a sensibilização, informações sobre sintomas, entrevistas com especialistas para alertar de maneira mais eficaz a comunidade, foi o período de maiores temperaturas, que corresponde de dezembro a abril.

Foi possível verificar (Figura 2) um declínio das ações de divulgação no meio do ano, nitidamente no mês de agosto em que foram contabilizadas apenas 18 publicações, em média de dois materiais midiáticos por ano. Na década analisada destaca-se que os meios de comunicação foram pautados pela demanda devido à incidência dos casos de dengue que foram notificados durante o período avaliado. As produções foram mais constantes entre dezembro e abril, que concentram o período de maior intensificação das ações da VAS. Neste período também se evidenciou maior envolvimento de outros setores públicos e da sociedade em atividades intersetoriais junto com a VAS. 
Figura 2 - Sazonalidade das ações e atividades desenvolvidas pela Vigilância Ambiental em Saúde de Chapecó, divulgadas e registradas pela Assessoria de Imprensa do município, no período de 2008 a 2017. As barras verticais representam o desvio padrão.

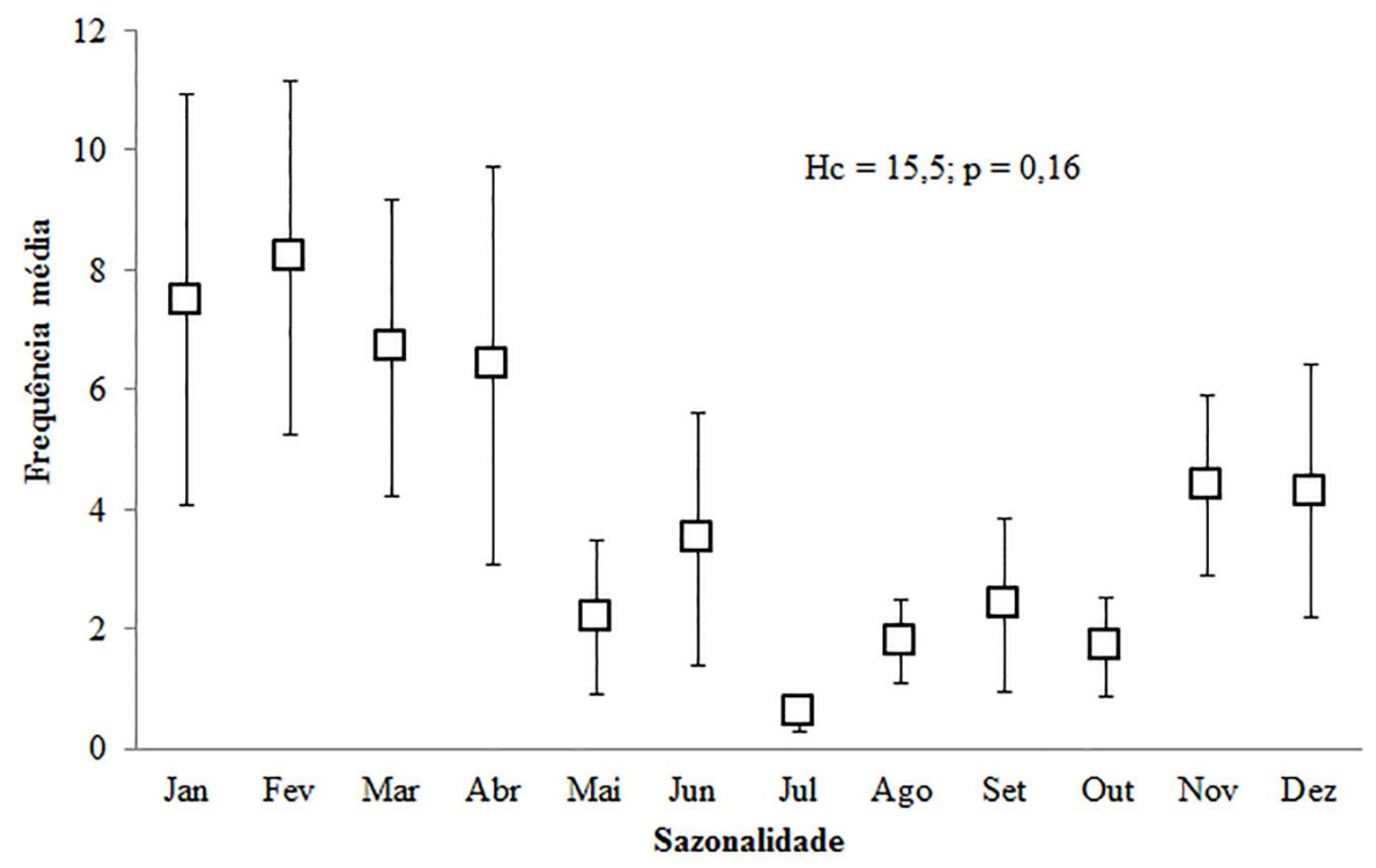

Fonte: Autores.

A divulgação de ações e campanhas de combate a endemias, vacinação, dentre outras, para informação, e possível mudança de comportamento da população, são uma constante do dos serviços de saúde no Brasil. Contudo, atingir e mudar comportamento tem sido um desafio e pode estar vinculado à falta representatividade no que se refere às imagens, linguagem, personagens e locais escolhidos e, até a superficialidade e rapidez dos materiais produzidos devido ao alto custo para veiculação ${ }^{17}$. Possivelmente, as produções locais, a exemplo do que está apresentado neste estudo, complementem e cheguem à população com maior sentido de pertencimento, por se tratar de situações dos lócus dessa população.

\section{Considerações Finais}

O estudo evidencia a importância da comunicação em saúde voltada às ações de promoção da saúde e na prevenção de agravos. Relata uma vasta parceria entre o serviço de VAS no município de Chapecó com vários setores públicos e da sociedade organizada. Configura-se em um resgate histórico da construção da VAS no município, dando destaque para as ações implementadas e desenvolvidas por esse serviço de saúde. Destaca a sazonalidade das ações, bem como a evolução ao longo de uma década. Sobretudo, tem o mérito de lançar luz sobre o papel da comunicação em saúde, com destaque para o trabalho profissional da Assessoria de Imprensa do município de Chapecó na produção, divulgação e registros das ações desenvolvidas pelo setor da VAS. 


\section{Agradecimentos}

À prefeitura de Chapecó-SC, pelo acesso aos dados. À Universidade Comunitária da Região de Chapecó (Unochapecó) pelo apoio à pesquisa e à produção científica. Ao Programa de Bolsas Universitárias de Santa Catarina (UNIEDU).

\section{Referências}

Albarado, A. J., Prado, E. J. \& Mendonça, A. V. M. (2019). Um, dois, três-gravando: as campanhas audiovisuais do Ministério da Saúde sobre dengue, chikungunya e Zika de 2014 a 2017. RECIIS - Revista Eletrônica de Comunicação, Informação e Inovação em Saúde, 13(1), 1-12.

Akermann, M. Sá, R. F., Moyses, S., Rezende, R. \& Rocha, D. (2014). Intersetorialidade? IntersetorialidadeS! Ciência e Saúde coletiva, 19(11): 4291-4300.

Augusto, L. G. S. (2004). Saúde e ambiente. In: Brasil. Ministério da Saúde. Saúde no Brasil: contribuições para a agenda de prioridades de pesquisa. Brasília, DF: Ministério da Saúde, (pp. 221-254). Ministério da Saúde.

Brasil. (1990). Lei No 8.080, de 19 de setembro de 1990. Lei orgânica da saúde: dispõe sobre as condições para a promoção, proteção e recuperação da saúde, a organização e o funcionamento dos serviços correspondentes e dá outras providências. Diário Oficial União. 20 set 1990. http://www.planalto.gov.br/ccivil_03/leis/L8080.htm.

Brasil. (2002). Ministério da Saúde. Fundação Nacional de Saúde. Vigilância Ambiental em Saúde. 46 p.

Brasil. Ministério da Educação. Secretaria de Educação Superior. (2001). Diretrizes Curriculares para os Cursos de Graduação. CNE/CES 492/2001. http://portal.mec.gov.br/cne/arquivos/pdf/CES0492.pdf.

Busato, M. A., Antoniolli, M. A. \& Ferraz, L. (2013). Interface saúde e ambiente. In: De Sá, C., Ferretti, F. \& Busato, M. A. (Eds.), Ensaios contemporâneos em saúde: uma perspectiva interdisciplinar (pp. 47-62). Chapecó, Santa Catarina/Brasil: Editora Argos.

Busato, M. A., Nothaft, S. C. S. \& Andrioli, D. C. (2018). Vigilância ambiental em saúde: aproximações possíveis com a educação em saúde e a promoção da saúde. In: Lutinski, J. A. \& Busato, M. A. (Eds.), Vigilância Ambiental em Saúde: uma abordagem interdisciplinar (pp. 167-179). Curitiba, Paraná/Brasil: CRV.

Chapecó. Estado de Santa Catarina. Prefeitura Municipal de Chapecó. (2015). Secretaria de Desenvolvimento Rural e Meio Ambiente. Plano de Desenvolvimento Rural. Chapecó.

Costa, L. O. F. C., Trindade, C. B. S., Ferreira, I. P. \& Nunes, E. C. R. (2021). Vigilância em Saúde \& planejamento e avaliação em Unidades de Saúde da Família: estudo qualitativo. Research, Society and Development, 10(6), e60010616275.

Franco, A. S. A. C., Lima, T. R. S. R. Clébicar, T. \& Aguiar, A. C. (2016). Saúde e meio ambiente nos currículos dos cursos de graduação em comunicação social no Brasil. RECIIS - Revista Eletrônica de Comunicação, Informação e Inovação em Saúde, 10(4), 10.29397/reciis.v10i4.1134.

Gómez, C. M. \& Minayo, M. C. S. (2006). Enfoque Ecossistêmico de Saúde e Qualidade de Saúde e ambiente sustentável: estreitando nós, Interface, 1(1), 119.

Hammer, O., Harper, D. A. T. \& Rian, P. D. (2001). Past: Palaeonthological statistics software package for education and data analysis. Version 1.37, http://palaeo-electronica.org/2001_1/past/issue1_01.htm

IBGE. Instituto Brasileiro de Geografia e Estatística (2020). Cidades: Chapecó, Santa Catarina. Brasil. https://cidades.ibge.gov.br/brasil/sc/chapeco/panorama.

Lutinski, J. A., Guarda, C. \& Lutinski, C. J. (2018). Vigilância Ambiental em Saúde em Chapecó-SC: experiências de uma década In: Lutinski, J. A \& Busato, M. A. (Eds.), Vigilância Ambiental em Saúde: uma abordagem interdisciplinar, (pp. 29-48). Curitiba, Paraná/Brasil, CRV.

Montoro, T. (2008). Retratos da comunicação em saúde: desafios e perspectivas. Interface, 12(25), 445-448.

Pessoni, A. \& Passaro, T. (2021). Os 12 eixos da comunicação combinada para a saúde pública municipal: uma proposta. Reciis - Revista Eletrônica de Comunicação, Informação \& Inovação em Saúde, 15(1), 155-171.

Santos, B. P., Feijó, A. M., Viegas, A. C. \& Lise, F. (2018). Classificação das pesquisas. In: Etapas da construção científica: da curiosidade acadêmica à publicação dos resultados (p.p. 139, 2018). Lise, F. F., Scwartz, B. M. \& Garcia, F. R. M. (Eds.), Pelotas, Rio Grande do Sul/Brasil: Ed. UFPel.

Silva, M. S. C. S., Junges, J. R., L'Abbate S. \& Moehlecke, V. (2021). Institutional analysis of health surveillance in a city in the Metropolitan Region of Porto Alegre, Rio Grande do Sul, Brazil. Saúde Sociedade. 30(1), e190904.

Ventura, A. N. G. F., Alencar, R. M., Araújo, I. S. \& Pinheiro, W. R. (2019). A Estratégia de Saúde da Família e o diálogo sobre a Intersetorialidade. Revista De Psicologia, 13(47), 63-76.

Villela, E. F. M. (2016). Comunicação de risco versus comunicação de crise na saúde pública: o discurso das autoridades diante de uma epidemia de dengue. RECIIS - Revista Eletrônica de Comunicação, Informação e Inovação em Saúde, 4(10), 1-11. 